\title{
On the evaluation quadratic forces on stationary bodies
}

\author{
Chang-Ho Lee \\ WAMIT Inc., Chestnut Hill MA, USA
}

June 9, 2006

\begin{abstract}
Conservation of momentum is applied to finite fluid volume surrounding a body and enclosed by the control surface in order to obtain expressions for all components of quadratic forces and moments acting on the body in terms of the momentum flux and the change of the momentum in the fluid volume. It is shown that the expressions derived are essentially identical with those obtained by a complementary approach in Chen(2005) where the pressure integrals on the body surface are tranformed into the integrals on the control surface using various vector theorems. Computational results are presented limited to the mean drift forces to illustrate the advantages of using control surfaces.
\end{abstract}

Keywords: drift force, quadratic force, control surface

\section{Introduction}

The second-order quadratic forces contribute to the excitation at low or high frequencies than those of incident waves which may be important for the analysis of structures with certain resonance features such as moored vessels and Tension Leg Platforms. They are also important for the analysis of drift motion of vessels which can be of particular concern when the vessels operate in the proximity of other structures. For certain structures such as ships and spars, it is of interest to have accurate prediction of slowly varying roll and pitch loads.

The quadratic forces can be evaluated by the integration of fluid pressure over the instantaneous wetted surface as shown in (Pinster, 1980), (Ogilvie, 1983), (Molin and Hairault, 1983) or (Lee and Newman, 1991). As a special case, the horizontal mean drift force and vertical moment can also be evaluated from the momentum conservation principle applied to the entire volume of fluid as shown in (Maruo, 1960) and (Newman, 1967). Other than this special case, the computational result of the quadratic pressure forces is generally less accurate than that of the first order forces. Thus it requires significantly more refined descritization entailing increased computing time. This is because of the evaluation of the fluid velocity, which contributes to the quadratic forces, is less accurate than the pressure on the body surface. When the body has sharp corners, the quadratic pressure near the corner is singular, though integrable, and it renders the computational result significantly inaccurate. Nonuniform discretization near the corner (Lee

(c) 2006 Kluwer Academic Publishers. Printed in the Netherlands. 
and Newman, 1992) in the low order method or nonuniform mapping in the higher order method (Lee, Farina, and Newman, 1998) do produce more accurate results than otherwise. However the computational results can still be inaccurate especially when the bodies experience large motion.

In order to overcome this difficulty, (Ferreira and Lee, 1994) applied momentum conservation over finite fluid volume surrounding the structures. All components of mean drift forces and moments on the body are obtained from the momentum flux through the control surface enclosing the fluid volume without the hydrodynamic pressure integration over the body surface. The computational results are significantly more accurate than the pressure integration. Recently (Chen, 2005) derived expressions for the quadratic forces and moments by transforming the pressure integration over the body surface into those on the control surface. One obvious advantage of these expressions is that the fluid velocity is not required on the body surface when body is fixed. Also the quadratic of the fluid velocity, which is most singular when body has sharp corners, in the pressure integration is not present in the new expressions having only linear terms in the fluid velocity.

In the following, we consider the conservation of momentum in the finite fluid volume surrounding a body and obtain the expressions for all components of quadratic forces and moments including complete mean drift forces and moments considered in (Ferreira and Lee, 1994). It is shown that these expressions are equivalent to those obtained by a complementary approach in (Chen, 2005). Computational results are presented for the mean drift forces to illustrate the advantage of current expressions.

\section{Formulation}

A potential flow is assumed which is governed by the velocity potential $\Phi(\vec{x}, t)$. The fluid pressure follows from Bernoulli's equation in the form

$$
p(\vec{x}, t)=-\rho\left(\Phi_{t}+\frac{1}{2} \nabla \Phi \cdot \nabla \Phi+g z\right)
$$

where $\rho$ is the fluid density and $g$ is gravity. $\vec{x}=(x, y, z)$ is the co-

ordinates in a space-fixed Cartesian coordinate system with positive $z$ pointing upward, perpendicular to the undisturbed free surface. $t$ denotes time.

The forces on the body are then obtained from

$$
\vec{F}=-\rho \iint_{s_{b}} \vec{n}\left[\Phi_{t}+\frac{1}{2} \nabla \Phi \cdot \nabla \Phi+g z\right] d s
$$


and the moment from

$$
\vec{M}=-\rho \iint_{s_{b}}(\vec{x} \times \vec{n})\left[\Phi_{t}+\frac{1}{2} \nabla \Phi \cdot \nabla \Phi+g z\right] d s
$$

where $\vec{n}$ is unit normal vector pointing outward from the fluid domain and $s_{b}$ denotes instantaneous wetted body surface.

The control volume considered is surrounded by $s_{b}$ and by the control surface $s_{c}$. If $s_{b}$ and $s_{c}$ intersect the free surface, we denote the intersection as $w$ and $c$, respectively. The free surface between $w$ and $c$ is denoted by $s_{f}$. It is assumed that $s_{b}$ and $s_{c}$ intersect the undisturbed free surface perpendicularly. The rate of change of the linear momentum $\mathbf{P}$ of the fluid in the control volume is

$$
\frac{d \mathbf{P}(t)}{d t}=\rho \frac{d}{d t} \iiint \vec{V} d v=\rho \iint_{s_{b f c}}\left[\Phi_{t} \vec{n}+\nabla \Phi(\vec{U} \cdot \vec{n})\right] d s
$$

and the rate of change of the angular momentum $\mathbf{H}$ is

$$
\begin{aligned}
\frac{d \mathbf{H}(t)}{d t} & =\rho \frac{d}{d t} \iiint(\vec{x} \times \vec{V}) d v \\
& =\rho \iint_{S_{b f c}}\left[\Phi_{t}(\vec{x} \times \vec{n})+(\vec{x} \times \nabla \Phi)(\vec{U} \cdot \vec{n})\right] d s
\end{aligned}
$$

Here $\vec{V}$ is the fluid velocity and $\vec{U}$ is the velocity of the control surface. Thus $\vec{U} \cdot \vec{n}=0$ on $s_{c}$ and $\vec{U} \cdot \vec{n}=\frac{\partial \Phi}{\partial n}$ on $s_{b}$ and $s_{f}$.

Using an identity given in (Newman, 1980, p134)

$$
\iint_{s_{b f c}}\left[\frac{\partial \Phi}{\partial n} \nabla \Phi-\frac{1}{2}(\nabla \Phi \cdot \nabla \Phi) \vec{n}\right] d s=0
$$

and the equations (4) and (5), we have the force and moment in the forms

$$
\begin{aligned}
\vec{F} & =\rho \iint_{s_{c}}\left[\left(\Phi_{t}+\frac{1}{2} \nabla \Phi \cdot \nabla \Phi\right) \vec{n}-\frac{\partial \Phi}{\partial n} \nabla \Phi\right] d s \\
& +\rho \iint_{s_{f}}\left(\Phi_{t}+\frac{1}{2} \nabla \Phi \cdot \nabla \Phi\right) \vec{n} d s-\rho g \iint_{S_{b}} z \vec{n} d s-\frac{d \mathbf{P}}{d t}
\end{aligned}
$$

and

$$
\begin{aligned}
\vec{M} & =\rho \iint_{s_{c}}\left[\left(\Phi_{t}+\frac{1}{2} \nabla \Phi \cdot \nabla \Phi\right)(\vec{x} \times \vec{n})-\frac{\partial \Phi}{\partial n}(\vec{x} \times \nabla \Phi)\right] d s \\
& +\rho \iint_{s_{f}}\left(\Phi_{t}+\frac{1}{2} \nabla \Phi \cdot \nabla \Phi\right)(\vec{x} \times \vec{n}) d s \\
& -\rho g \iint_{S_{b}} z(\vec{x} \times \vec{n}) d s-\frac{d \mathbf{H}}{d t}
\end{aligned}
$$


Considering quadratic terms from the foregoing equations as shown in Appendix, we have the expressions for the quadratic forces and moments. We first consider the mean drift forces and moments. Since the time averages of last terms in the equations (7) and (8) vanish, there is no contribution from these terms to the mean forces and moments. The force can be obtained from the time average of

$$
\begin{aligned}
\vec{F}^{(2)}= & -\frac{1}{2} \frac{\rho}{g} \int_{C} \vec{n}^{\prime} \phi_{t}^{2} d l-\rho g \int_{W}\left[\zeta\left(\vec{\Xi} \cdot \vec{n}^{\prime}\right)\right] \vec{k} d l \\
& -\rho \iint_{S_{c}}\left[\nabla \phi \frac{\partial \phi}{\partial n}-\frac{1}{2} \vec{n}(\nabla \phi \cdot \nabla \phi)\right] d s \\
& +\rho \vec{k} \iint_{S_{f}}\left(\zeta \frac{\partial \phi_{t}}{\partial z}+\frac{1}{2} \nabla \phi \cdot \nabla \phi\right) d s+\vec{F}_{S}^{(2)}
\end{aligned}
$$

and the moment from

$$
\begin{aligned}
\vec{M}^{(2)}= & -\frac{1}{2} \frac{\rho}{g} \int_{C}\left(\vec{x} \times \vec{n}^{\prime}\right) \phi_{t}^{2} d l-\rho g \int_{W} \zeta\left(\vec{\Xi} \cdot \vec{n}^{\prime}\right)(\vec{x} \times \vec{k}) d l \\
& -\rho \iint_{S_{c}}\left[(\vec{x} \times \nabla \phi) \frac{\partial \phi}{\partial n}-\frac{1}{2}(\vec{x} \times \vec{n})(\nabla \phi \cdot \nabla \phi)\right] d s \\
& +\rho \iint_{S_{f}}(\vec{x} \times \vec{k})\left(\zeta \frac{\partial \phi_{t}}{\partial z}+\frac{1}{2} \nabla \phi \cdot \nabla \phi\right) d s+\vec{M}_{S}^{(2)}
\end{aligned}
$$

Here $\phi$ denotes the first order velocity potential and $\zeta=-(1 / g) \phi_{t}$ denotes the first order wave elevation. $S_{b}, S_{f}$ and $S_{c}$ are undisturbed body surface, free surface and control surface. $W$ and $C$ are the intersections of $S_{b}$ and $S_{c}$ with undisturbed free surface. $\vec{n}^{\prime}$ denotes two dimensional normal vector to $W$ and $C$ on $S_{f}, \nabla^{\prime}$ two dimensional gradient on $S_{f}$ and $\vec{k}$ the unit vector in $z . \vec{\Xi}=\left(\Xi_{1}, \Xi_{2}, \Xi_{3}\right)=\vec{\xi}+\vec{\alpha} \times \vec{x}$ where $\vec{\xi}$ and $\vec{\alpha}$ denote the motion amplitudes of the translational and the rotational modes, respectively. Finally $\vec{F}_{S}^{(2)}$ and $\vec{M}_{S}^{(2)}$ denote parts of hydrostatic forces and moments and they are given in Appendix. We note above equations are different from those in (Ferreira and Lee, 1994).

The expressions for the quadratic forces and moments are completed by adding the changes of the linear momentum of the fluid volume

$$
\begin{aligned}
-\frac{d \mathbf{P}}{d t}= & -\rho \iint_{S_{f}}\left[\nabla \phi \frac{\partial \phi}{\partial n}+\zeta \nabla \phi_{t}\right] d s \\
& -\rho \iint_{S_{b}}\left[\nabla \phi\left(\frac{d \vec{\Xi}}{d t} \cdot \vec{n}\right)+(\Xi \cdot \vec{n}) \nabla \phi_{t}\right] d s
\end{aligned}
$$

and the angular momentum

$$
-\frac{d \mathbf{H}}{d t}=-\rho \iint_{S_{f}} \mathbf{x} \times\left[\nabla \phi \frac{\partial \phi}{\partial n}+\zeta \nabla \phi_{t}\right] d s
$$




$$
-\rho \iint_{S_{b}} \mathbf{x} \times\left[\nabla \phi\left(\frac{d \vec{\Xi}}{d t} \cdot \vec{n}\right)+(\Xi \cdot \vec{n}) \nabla \phi_{t}\right] d s
$$

to the equations (9) and (10). Among several expressions, (11) and (12) render the total forces and moments in the most compact forms. The derivations of these equations are provided in the Appendix. The final expressions for the quadratic forces and moments derived here are the same as those given in (Chen, 2005).

\section{Numerical Results and Discussions}

We first consider a hemisphere which is freely floating in infinite water depth. The incident wave travels to the positive $x$ axis. Figure 1 shows the hemisphere enclosed by the cylindrical control surface. The radius of the sphere is 1 meter and the radius and draft of the control surface are 1.2 meters. Computations are made using the higher-order option of the panel program (WAMIT, 2005). The geometry of the sphere and that of the control surface are represented analytically. A quadrant of the hemisphere is represented by a patch and a quadrant of the interior free surface of the hemisphere is also represented by a patch. The latter is introduced to eliminate the effect of the irregular frequencies. The unknown velocity potential on each patch is represented by quadratic B-splines. Each patch is subdivided into 1, 4 and 16 higher order panels to examine the convergence of the computational results. On the control surface, a fixed number of control points in the calculation of the momentum flux. The bottom, side and top of the cylindrical control surface are represented by 12, 12 and 4 subdivisions, respectively. The integration is carried out using 9 nodes Gauss quadrature on each subdivision assuming quadratic variation of the momentum flux. Thus 252 control points are used in total. The mean surge drift forces on the hemisphere are showed on the left column of Figure 3 which will be discussed below.

Next we consider a freely floating truncated circular cylinder of radius and draft 1 meters in infinite water depth. The center of rotation of the cylinder is at the intersection of the axis of the cylinder with the free surface while the center of gravity is 1 meter below the free surface. The radius of gyration of the pitch mode is 0.5 meters. Figure 2 shows the cylinder and the control surface. Three patches are used to represented the cylinder including the interior free surface and 3, 12 and 48 higher-order panels are used in the computation. The geometry of the cylinder is represented analytically with nonuniform mapping near the corner. As in the previous computation, 252 control points 
are used, in total, on the same cylindrical control surface of the radius and draft of 1.2 meters. The mean surge drift forces on the cylinder are showed on the right column of Figure 3.

Figure 3 shows the surge mean drift forces on the hemisphere on the left column and those on the cylinder on the right column. The computational results are more accurate toward the bottom plots for which finer discretization is used. Each plot contains three surge forces computed by three approaches; the pressure integration on the body surface (Lee and Newman, 1991), the far field momentum conservation (Maruo, 1960)(Newman, 1967) and the momentum conservation within the control surface. The figure shows the results from the pressure integration are least accurate. Specifically, while the mean surge force on the cylinder, which has a sharp corner, can be calculated accurately using 3 panels up to around $K R=3$ by momentum conservation, it is necessary to use 48 panels for the pressure integration. Since the computational time for the linear solution in the higher-order method is typically proportional to the square of the number of panels, the momentum conservation can be orders of magnitude more efficient than the pressure integration for the evaluation of the mean forces. The figure also shows the results using the control surface are identical with those from the momentum conservation to the graphical accuracy. The computational time using the control surface depends on the number of control points. Using compact control surfaces surrounding the body, as shown in this example, the additional computating time for the calculation of the momentum flux on the control surface can be similar to that for the linear solution.

This example illustrates the advantages of using control surface for the calculation of mean forces. The computational results are as accurate as those from the far field momentum conservation. All components

of mean forces and moments can be calculated more efficiently than the pressure integration. For multiple bodies, the forces and moments on individual body can be obtained using separate control surface surrounding each body which is not possible by the far field momentum conservation.

\section{Conclusion}

We derived expressions for the quadratic forces and moments by applying momentum conservation in the finite volume surrounding the body. The final form of the expressions can be made to be identical to those obtained by (Chen, 2005). Computations of mean drift forces show the accuracy and efficiency of using the control surfaces. All compo- 


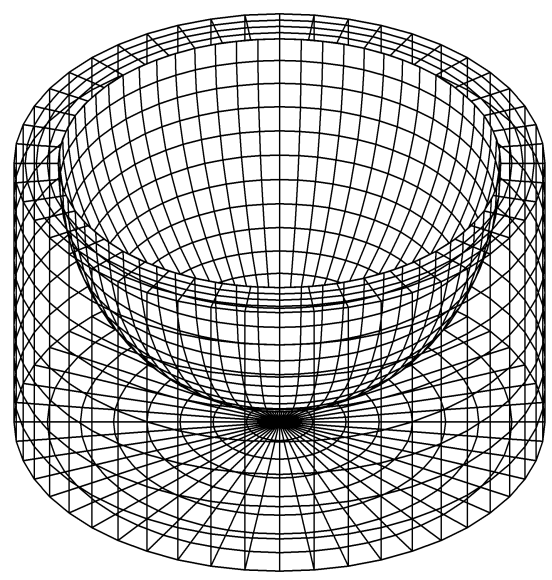

Figure 1. Geometry of the hemisphere and control surface. The radius of the sphere is 1 . The radius and draft of the cylindrical control surface are 1.2. The meshes are for the purpose of the visualization only.

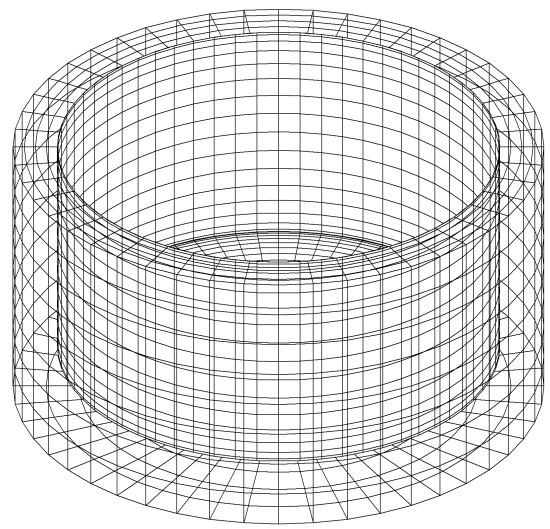

Figure 2. Geometry of the cylinder and control surface. The radius and draft of the cylinder are 1 . The radius and draft of the cylindrical control surface are 1.2. The meshes are for the purpose of the visualization only. 

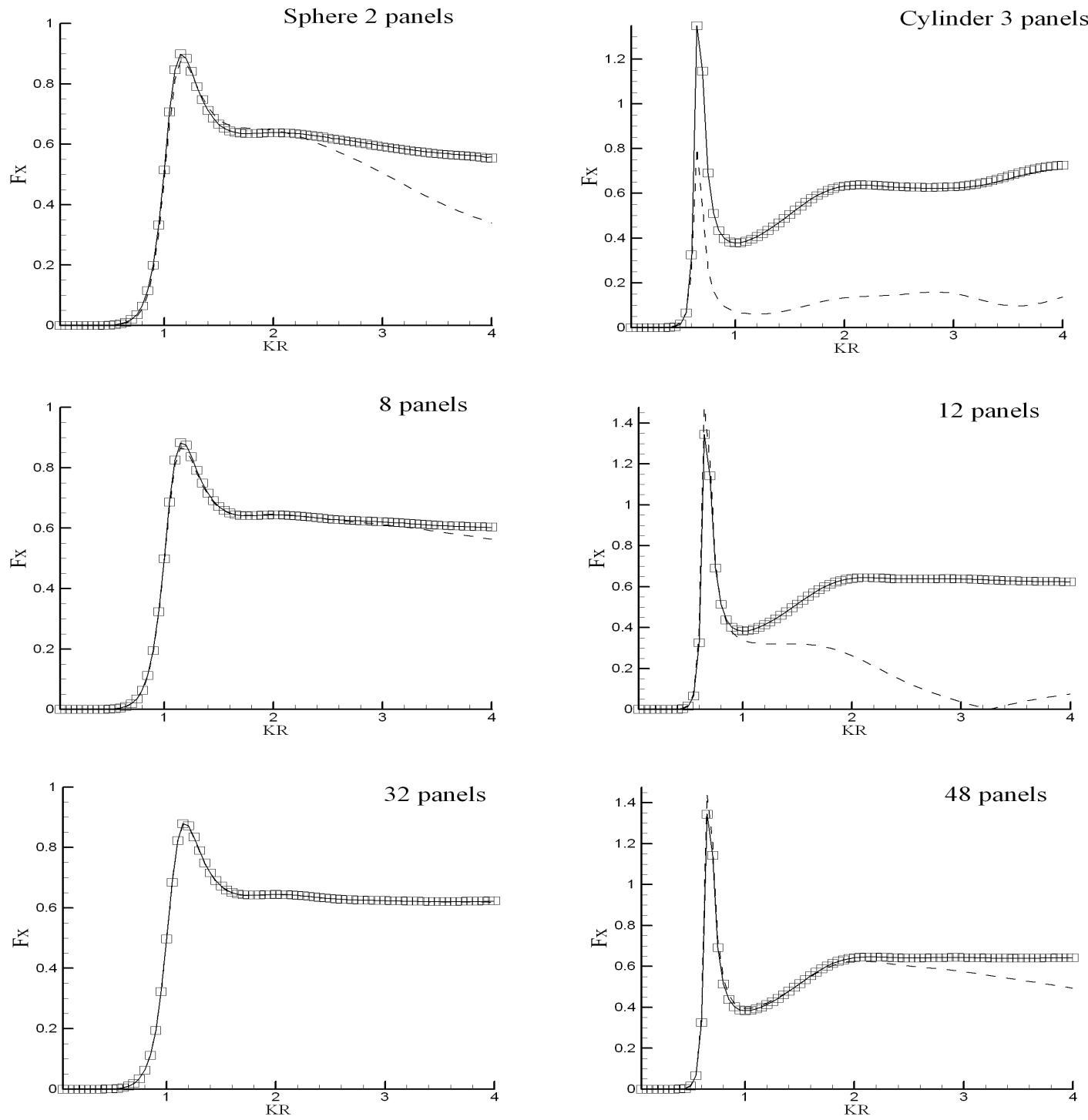

Figure 3. Nondimensional mean surge forces on the hemisphere and cylinder. The forces on the hemisphere is on the left column and those on the cylinder on the right column. The forces are normalized by $\rho g R A^{2}$ where $\rho$ is the water density, $g$ is the gravitational acceleration, $R$ is the radius and $A$ is the wave amplitude. $K$ is the infinite depth wave number. Forces by the pressure integration are represented by dashed lines, those by the momentum conservation by solid lines and those by using control surface are represented by squares. 
nents of the forces and moments can be evaluated as with the pressure integration but by avoiding the integration of pressure on the body the computational results are as accurate as the far field momentum conservation.

The expressions for the quadratic forces and moments in bichromatic waves contain the integration over the body surface of the pressure proportional to the fluid velocity, as shown in the equations (11) and (12). Thus further study is needed to find the computational advantage of the current approach, in particular, when the body has sharp corners. However, in comparison with the pressure integration, the pressure to be integrated is less singular. In addition, when low frequency forces are of interest, the contribution from the integration over the body surface will be small, linearly proportional to the difference of two frequencies.

\section{Appendix}

The quadratic terms of the integral on $s_{c}$, denoted by $F_{S_{c}}$, are given in the form

$$
\vec{F}_{S_{c}}^{(2)}=-\frac{\rho}{g} \int_{C} \vec{n}^{\prime} \phi_{t}^{2} d l-\rho \iint_{S_{c}}\left[\nabla \phi \frac{\partial \phi}{\partial n}-\frac{1}{2} \vec{n}(\nabla \phi \cdot \nabla \phi)\right] d s
$$

where the first line integral accounts for the momentum flux over the portion of $s_{c}$ for $z=(0, \zeta)$.

The quadratic terms of the integral on $s_{f}$, denoted by $F_{S_{f}}$, are

$$
\begin{aligned}
\vec{F}_{S_{f}}^{(2)}= & -\rho g \vec{k} \int_{W}\left[\zeta\left(\vec{\Xi} \cdot \vec{n}^{\prime}\right)\right] d l+\frac{\rho \vec{k}}{2} \iint_{S_{f}}(\nabla \phi \cdot \nabla \phi) d s \\
& +\frac{\rho}{g} \iint_{S_{f}} \phi_{t} \nabla^{\prime} \phi_{t} d s+\rho \vec{k} \iint_{S_{f}} \zeta \frac{\partial \phi_{t}}{\partial z} d s
\end{aligned}
$$

where the first line integral accounts for the vertical momentum flux over the portion of free surface between the mean position of the waterline $W$ and the unsteady line of intersection of the body with the free surface $w$. The third integral accounts for the horizontal momentum flux due to the slope of the free surface elevation. This term was omitted from the equation (13) in (Ferreira and Lee, 1994). The last integral is due to the expansion of the velocity potential from $S_{f}$ to $\zeta$.

The quadratic terms due to the hydrostatic pressure on $s_{b}$ are obtained by two integrals. One is over the mean wetted body surface $S_{b}$ and the result, following (Lee and Newman, 1991), takes a form

$$
\begin{aligned}
\vec{F}_{S_{b}}^{(2)} & =-\rho g \iint_{S_{b}} z \vec{n} d s \\
& =\vec{\alpha} \times\left(-\rho g A_{w p}\left(\xi_{3}+\alpha_{1} y_{f}-\alpha_{2} x_{f}\right) \vec{k}\right)+F_{S}^{(2)}
\end{aligned}
$$


where $A_{w p}$ is the waterplane area, $x_{f}$ and $y_{f}$ are the coordinates of the center of floatation and

$$
F_{S}^{(2)}=-\rho g A_{w p}\left[\alpha_{1} \alpha_{3} x_{f}+\alpha_{2} \alpha_{3} y_{f}+\frac{1}{2}\left(\alpha_{1}^{2}+\alpha_{2}^{2}\right) Z_{o}\right] \vec{k}
$$

Here $Z_{o}$ denotes the vertical coodinate of the origin of the body-fixed coordinates system relative to the mean free surface.

The second integral is over the region between $z=\Xi_{3}$ and $z=\zeta$ on $s_{b}$ and it takes the form

$$
\vec{F}_{W}^{(2)}-\rho g \int_{W} d l \int_{0}^{\zeta-\Xi_{3}}\left(\bar{z}+\Xi_{3}\right) \vec{n} d \bar{z}=-\frac{1}{2} \rho g\left(\int_{W} \vec{n}^{\prime} \zeta^{2} d l-\int_{W} \vec{n}^{\prime} \Xi_{3}^{2} d l(17)\right.
$$

where the vertical coordinate of the body fixed coordinates system $\bar{z}=z-\Xi_{3}$.

Invoking Stoke's theorem to a vector $\vec{V}$, we have a relation

$$
\iint[(\vec{n} \times \nabla) \times \vec{V}] d s=\int(\vec{t} \times \vec{V}) d l=-\int\left[V_{3} \vec{n}^{\prime}-\left(\vec{V} \cdot \vec{n}^{\prime}\right) \vec{k}\right] d l
$$

when the tangential vector $t$ in the line integral is perpendicular to $\vec{k}$ Applying this relation over the region enclosed by the waterline, $W$ with $\vec{V}=\left(0,0, \Xi_{3}^{2}\right)$, we have

$$
\frac{1}{2} \rho g \int_{W}\left(\vec{n}^{\prime} \Xi_{3}^{2}\right) d l=-\vec{\alpha} \times\left(-\rho g A_{w p}\left(\xi_{3}+\alpha_{1} y_{f}-\alpha_{2} x_{f}\right) \vec{k}\right)
$$

Similarly, applying this relation between $W$ and $C$ with $\vec{V}=\left(0,0, \zeta^{2}\right)$, we have

$$
\frac{1}{2} \rho g\left(\int_{W} \vec{n}^{\prime} \zeta^{2} d l+\int_{C} \vec{n}^{\prime} \zeta^{2} d l\right)=\frac{\rho}{g} \iint_{S_{f}} \phi_{t} \nabla^{\prime} \phi_{t} d s
$$

Excluding the change of momentum $d \mathbf{P} / d t$, the quadratic forces can be obtained as the sum of $\vec{F}_{S_{c}}^{(2)}, \vec{F}_{S_{f}}^{(2)}, \vec{F}_{S_{b}}^{(2)}$ and $\vec{F}_{W}^{(2)}$. Upon substituting the relations in the equations (19) and (20) to this sum, we have the mean forces in the form shown in the equation (9). The expression for the moments can be obtained in the similar manner and it is shown in the equation (10). Here we provide the hydrostatic moments, $M_{S}^{(2)}$, for the completeness.

$$
\begin{aligned}
M_{S}^{(2)} & =\rho g\left\{\left[-A_{w p}\left(\xi_{3} \alpha_{3} x_{f}+\frac{1}{2}\left(\alpha_{1}^{2}+\alpha_{2}^{2}\right) Z_{o} y_{f}\right)-2 \alpha_{1} \alpha_{3} L_{12}\right.\right. \\
& \left.+\alpha_{2} \alpha_{3}\left(L_{11}-L_{22}\right)+\forall\left(\alpha_{1} \alpha_{2} x_{b}-\frac{1}{2}\left(\alpha_{1}^{2}+\alpha_{3}^{2}\right) y_{b}\right)\right] \\
& \left.-A_{w p}\left(\xi_{3}+\alpha_{1} y_{f}-\alpha_{2} x_{f}\right)\left(\alpha_{1} Z_{o}+\xi_{2}\right)\right\} \vec{i}
\end{aligned}
$$




$$
\begin{aligned}
& +\rho g\left\{\left[-A_{w p}\left(\xi_{3} \alpha_{3} y_{f}-\frac{1}{2}\left(\alpha_{1}^{2}+\alpha_{2}^{2}\right) Z_{o} x_{f}\right)+2 \alpha_{2} \alpha_{3} L_{12}\right.\right. \\
& \left.\left.+\alpha_{1} \alpha_{3}\left(L_{11}-L_{22}\right)+\forall \frac{1}{2}\left(\alpha_{2}^{2}+\alpha_{3}^{2}\right) x_{b}\right)\right] \\
& \left.-A_{w p}\left(\xi_{3}+\alpha_{1} y_{f}-\alpha_{2} x_{f}\right)\left(\alpha_{2} Z_{o}-\xi_{1}\right)\right\} \vec{j}
\end{aligned}
$$

where $\forall$ denotes the volume of the body and $x_{b}$ and $y_{b}$ are the coordinates of the center of buoyancy. $L_{i j}$ denotes the moments of the waterplane area with the subscript $i$ and $j$ corresponding to the $x$ and $y$ coordinates.

We next consider the quadratic terms due to the change of momentum inside the control volume in the equations (4) and (5). The quadratic term of the integral on $s_{c}$ vanishes. Those on $s_{b}$ and $s_{f}$ can be expressed in terms of the integrals over the mean surfaces, $S_{b}$ and $S_{f}$. Invoking Stoke's theorem on $S_{b}$ and using the vector relations given in the equations (74d) and (74e) of Chapter 6 of (Hildebrand, 1976) we have following two relations, one for the linear momentum

$$
\begin{gathered}
g \int_{W} \zeta\left[\left(\Xi_{3} \vec{n}^{\prime}-\left(\vec{\Xi} \cdot \vec{n}^{\prime}\right) \vec{k}\right] d l+\alpha \times \iint_{S_{b}} \phi_{t} \vec{n} d s\right. \\
=\iint_{S_{b}}\left[(\Xi \cdot \vec{n}) \nabla \phi_{t}-\vec{n}\left(\vec{\Xi} \cdot \nabla \phi_{t}\right)\right] d s
\end{gathered}
$$

and the other for the angular momentum

$$
\begin{gathered}
g \int_{W} \zeta \vec{x} \times\left[\Xi_{3} \vec{n}^{\prime}-\left(\vec{\Xi} \cdot \vec{n}^{\prime}\right) \vec{k}\right] d l+\vec{\xi} \times \iint_{S_{b}} \phi_{t} \vec{n} d s+\vec{\alpha} \times \iint_{S_{b}}(\vec{x} \times \vec{n}) \phi_{t} d s \\
=\iint_{S_{b}}\left[(\Xi \cdot \vec{n})\left(\vec{x} \times \nabla \phi_{t}\right)-(\vec{x} \times \vec{n})\left(\vec{\Xi} \cdot \nabla \phi_{t}\right)\right] d s
\end{gathered}
$$

Using above relations, it can be shown that the changes of momentum in (4) and (5) take the forms

$$
\begin{aligned}
\frac{d \mathbf{P}(t)}{d t} & =\rho \iint_{S_{f}}\left[\nabla \phi \frac{\partial \phi}{\partial n}+\zeta \nabla \phi_{t}\right] d s \\
& +\rho \iint_{S_{b}}\left[\nabla \phi\left(\frac{d \vec{\Xi}}{d t} \cdot \vec{n}\right)+(\Xi \cdot \vec{n}) \nabla \phi_{t}\right] d s
\end{aligned}
$$

and

$$
\begin{aligned}
\frac{d \mathbf{H}(t)}{d t} & =\rho \iint_{S_{f}} \mathbf{x} \times\left[\nabla \phi \frac{\partial \phi}{\partial n}+\zeta \nabla \phi_{t}\right] d s \\
& +\rho \iint_{S_{b}} \mathbf{x} \times\left[\nabla \phi\left(\frac{d \vec{\Xi}}{d t} \cdot \vec{n}\right)+(\Xi \cdot \vec{n}) \nabla \phi_{t}\right] d s
\end{aligned}
$$




\section{References}

Pinster, J. A. Low frequency second-order exciting forces on floating structures. NSMB report 6501980.

Ogilvie, T. F. Second-order hydrodynamic effects on ocean platforms. International Workshop on Ship and Platform Motions, Berkeley, 1983.

Molin, B. and J.-P. Hairault On Secon-Order Motion and Vertical Drift Forces for Three-Dimensional Bodies in Regular Waves. International Workshop on Ship and Platform Motions, Berkeley, 1983.

Lee, C.-H. and J. N. Newman First- and Second-order Wave Effects on a Submerged Spheroid. Journal of Ship Research, 35:183-190, 1991.

Maruo, H. The Drift of a Body Floating on Waves. Journal of Ship Research, 4:1-10, 1960.

Newman, J. N. The Drift Force and Moment on Ships in Waves. Journal of Ship Research, 11:51-60, 1967.

Ferreira, M. D. and C.-H. Lee Computation of Second-Order Mean Wave Forces and Moments in Multibody Interaction. Proceedings of Behaviour of Offshore Structures, 2:303-313, 1994.

Chen, X.-B. Computation of Low-Frequency Loads by the Middle-Field Formulation. 20th Workshop for Water Waves and Floating Bodies, 2005.

Lee, C.-H. and J. N. Newman Sensitivity of Wave Load to the Discretization of Bodies. Proceedings of Behaviour of Offshore Structures, 1992.

Lee, C.-H., L. Farina L., and J. N. Newman A Geometry-Independent HigherOrder Panel Method and its Application to WaveBody Interactions. Proceedings of Engineering Mathematics and Applications Conference, 303-306, Adelaide, 1998.

Newman, J. N. Marine Hydrodynamics. The Massachusetts Institute of Technology, 1980.

Hildebrand, F. B. Advanced Calculus for Engineers. Prentice-Hall, 1976.

WAMIT User Manual www.wamit.com, 2005. 